\title{
11-Deoxycortisol may be superior to cortisol in confirming a successful adrenal vein catheterization without cosyntropin: a pilot study
}

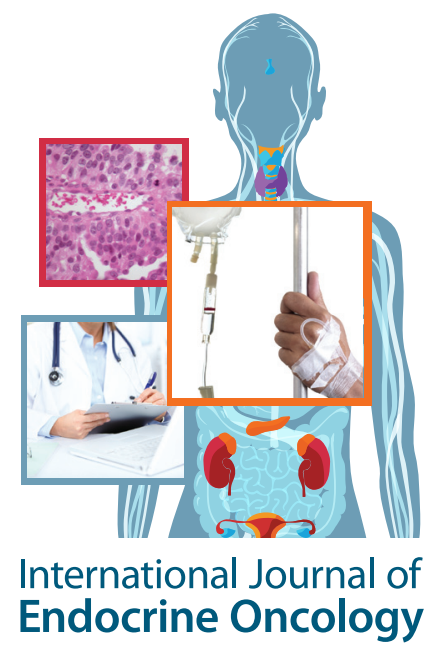

\author{
Naris Nilubol ${ }^{*, 1}$, Steven J Soldin ${ }^{2,4}$, Dhaval Patel', Muthoni Rwenji², Jianghong Gu' ${ }^{2}$, \\ Likhona S Masika², Richard Chang ${ }^{5}$, Constantine A Stratakis ${ }^{3} \&$ Electron Kebebew $^{1}$
}

\section{Practice points}

- Because imaging alone cannot reliably distinguish aldosterone-producing adenoma from commonly detected nonfunctioning adrenal nodules. Adrenal venous sampling is considered the gold standard for selecting patients who would benefit from an adrenalectomy.

- There are two areas that need to be assessed from adrenal venous sampling: the position of the catheter when the samples are collected and a comparison of normalized aldosterone production from each adrenal gland.

- To confirm a successful adrenal vein catheterization, a commonly used criterion is the ratio of cortisol from the adrenal vein to the peripheral vein greater than 3:1 without cosyntropin infusion.

- Compared with the cosyntropin-stimulated cortisol ratio of adrenal to peripheral veins, the nonstimulated ratio can be falsely low, suggesting incorrect catheter placement occurred in approximately $50 \%$ of the samples.

- Our pilot study suggests that 11-deoxycortisol is more accurate in identifying correct position of the catheter than cortisol without cosyntropin infusion. Thus, it can simplify the procedure and reduce false-negative results associated with cosyntropin infusion.

Aim: We aimed to compare the performance of nine adrenal steroids in confirming the correct catheter position during adrenal venous sampling (AVS) without cosyntropin in patients with primary hyperaldosteronism. Materials \& methods: A successful adrenal vein catheterization without cosyntropin was defined as the ratio of steroids from adrenal to peripheral veins being >3:1. AVS samples from four patients with primary hyperaldosteronism were analyzed. Results: Compared with the mean ratio of cortisol without cosyntropin, the ratios of 11-deoxycortisol $(p=0.008)$, dehydroepiandrosterone $(p=0.01)$ and androstenedione $(p=0.008)$ were significantly higher. None of the ratios $(n=8)$ of cortisol from adrenal to peripheral veins exceeded 3:1, while all ratios of 11-deoxycortisol $(p<0.001)$ were $>3$. Conclusion: Cosyntropin infusion during AVS may not be necessary if 11-deoxycortisol is used to confirm catheter position.

First draft submitted: 25 November 2016; Accepted for publication: 7 February 2017; Published online: 27 April 2017

'Endocrine Oncology Branch, National Cancer Institute, National Institutes of Health, MD 20892, USA

2Department of Laboratory Medicine, National Institutes of Health, MD 20892, USA

${ }^{3}$ Section on Endocrinology \& Genetics, Eunice Kennedy Shriver National Institute of Child Health \& Human Development, National

Institutes of Health, MD 20892, USA

${ }^{4}$ Department of Medicine, Division of Endocrinology \& Metabolism, Georgetown University, WA 20007, USA

${ }^{5}$ Endocrine \& Venous Services Section, Interventional Radiology Section, Radiology \& Imaging Sciences, National Institutes of Health, MD 20892, USA

*Author for correspondence: Tel.. +1 301451 2355; niluboln@mail.nih.gov

Future:
Medicine $\mathrm{fS}$ part of 


\section{KEYWORDS}

- 11-deoxycortisol

- adrenal vein sampling

- aldosterone $\cdot$ cortisol

- cosyntropin • primary

hyperaldosteronism
Primary hyperaldosteronism (PA) was first described in 1954 by Jerome W Conn, who reported a patient with hypertension, hypokalemia and a $4 \mathrm{~cm}$ aldosterone-producing adenoma [1]. PA is the most common cause of secondary hypertension, with a prevalence of $5-15 \%[2,3]$. The long-term effects of PA results in an increased incidence of cardiovascular events, independent of blood pressure [4]. Because more than $50 \%$ of patients with PA have bilateral adrenal disease [2], it is critically important to identify patients with unilateral disease that can be treated surgically. Although anatomical imaging, such as a CT scan or MRI, can reliably detect adrenal nodules larger than $1 \mathrm{~cm} \mathrm{[5],} \mathrm{imaging} \mathrm{alone} \mathrm{cannot} \mathrm{reliably} \mathrm{distin-}$ guish aldosterone-producing adenoma from commonly detected nonfunctioning adrenal nodules. Adrenal venous sampling (AVS) is considered the gold standard for selecting patients who would benefit from an adrenalectomy. We previously demonstrated that AVS altered the management in $50 \%$ of patients with PA by identifying unilateral hyperfunctioning adrenal glands in those with bilateral abnormalities or no abnormalities based on imaging studies. In addition, AVS identified contralateral hyperfunctioning adrenal glands and bilateral disease in those patients with a unilateral adrenal mass on imaging studies [6].

There are two areas that need to be assessed from AVS: the position of the catheter when the samples are collected and a comparison of normalized aldosterone production from each adrenal gland. There have been at least ten criteria describing the methods for confirming that the catheter is in the adrenal vein and identifying patients with unilateral disease. All criteria use cortisol to normalize the aldosterone level. The most accurate criterion for identifying and predicting PA lateralization is when the ratio of aldosterone normalized with cortisol from the hypersecreting gland is greater than fourtimes higher than the ratio of the contralateral gland after cosyntropin is administered [7]. To confirm a successful adrenal vein catheterization, a method widely used is comparing the ratio of cortisol from the adrenal vein to the peripheral vein. Cosyntropin is administered to the patient to increase the ratio of cortisol from adrenal to peripheral vein and confirm a successful adrenal vein catheterization. The adrenal to peripheral vein cortisol ratio is typically more than $10: 1$, with the continuous cosyntropin infusion protocol $[8]$ and more than 3:1 without the use of cosyntropin [9]. The criteria for biochemically confirming a successful adrenal vein catheterization remain debatable [10]. In some series, it is defined as the cortisol ratio of adrenal to peripheral veins $>2$ or $>3: 1[11,12]$, but the use of cosyntropin remains controversial [13,14]. The omission of cosyntropin may increase the biochemical failure rate of adrenal vein catheterization because of the lack of exaggerated cortisol levels. Compared with the cosyntropinstimulated cortisol ratio of adrenal to peripheral veins, the nonstimulated ratio can be falsely low, suggesting incorrect catheter placement occurred in approximately $50 \%$ of the samples when the cortisol ratio of adrenal to peripheral vein of 3:1 was used [15].

We hypothesized that other steroids can be used to confirm a successful adrenal vein catheterization without cosyntropin infusion, and can be used for lateralization. Thus, we simultaneously analyzed eight additional steroids in specimens obtained during AVS, before and after cosyntropin infusion, and compared the results to cortisol.

\section{Materials \& methods}

We selected four consecutive patients with PA who underwent AVS as per the clinical protocol approved by our Institutional Review Board. All patients provided written informed consent. Patient demographics and clinical data were obtained from a computerized medical record system. The diagnosis of PA was made based on an elevated serum aldosterone to plasma renin ratio. All patients underwent a confirmatory test with a sodium chloride loading test. Pheochromocytoma and hypercortisolemia were biochemically excluded. Pheochromocytoma was excluded by normal fractionated plasma or urinary metanephrines. Hypercortisolemia was excluded by a normal 24-h urinary free cortisol level and a suppressed cortisol after $1 \mathrm{mg}$ dexamethasone was given at night. None of the patients had chronic kidney disease. Patients with unilateral disease based on AVS results underwent a laparoscopic adrenalectomy. Biochemical remission was defined as postoperative aldosterone to renin ratio $<30$ with an aldosterone level $<15 \mathrm{ng} / \mathrm{ml}$.

\section{- AVS technique}

AVS was performed by an experienced interventional radiologist. The technique was as 
previously described [7]. In brief, bilateral adrenal veins were simultaneously catheterized via bilateral femoral veins. Peripheral samples were obtained from the right iliac vein. The placement of the catheters was confirmed radiographically using contrast venography and subsequently by an elevated ratio of cortisol from each adrenal to iliac vein. The baseline samples were obtained from each adrenal vein and iliac vein, followed by an infusion of $0.25 \mathrm{mg}$ of cosyntropin in $250 \mathrm{ml}$ of normal saline at a rate of $150 \mathrm{ml} / \mathrm{h}$. Blood samples were collected at 5, 10 and $15 \mathrm{~min}$ after cosyntropin was administered, and then cortisol and aldosterone levels were measured. For this study, we prospectively collected additional samples at baseline and at $10 \mathrm{~min}$ after cosyntropin infusion. Serum was isolated and stored at $-80^{\circ} \mathrm{C}$.

\section{- Interpretation of AVS results}

The criteria used to confirm a successful adrenal vein catheterization was the ratio of cortisol from adrenal to peripheral veins of $>3$ and $>5$, before and after cosyntropin infusion, respectively. The criterion for identifying patients with unilateral hyperfunctioning adrenal glands was the ratio of normalized aldosterone to cortisol levels from the hypersecreting gland being $>2$ and $>4$-times higher than the ratio from the contralateral adrenal gland without and with cosyntropin infusion, respectively [7].

\section{- Liquid chromatography-tandem mass spectrometry}

All steroid hormones were analyzed at the NIH Clinical Center (MD, USA). The liquid chromatography-tandem mass spectrometry (LCMS/MS) method was used to quantify nine steroids as described in our recent paper [16]. These steroids included cortisone, corticosterone, cortisol, 11-deoxycortisol, androstenedione, $17 \alpha$-hydroxyprogesterone (17-OHPG), testosterone, progesterone and dehydroepiandrosterone (DHEA).

Aldosterone and cortisol were analyzed on the DiaSorin Liason XL (DiaSorin, OK, USA) and Immulite $2000 \mathrm{XPi}$ (Siemens Medical Solutions USA, Inc., PA, USA), respectively. Immunoassays were performed according to the manufacturers' instructions.

Interassay variation was assessed by simultaneously analyzing cortisol levels, along with other steroid levels, and comparing those results to the results analyzed by a clinical laboratory.

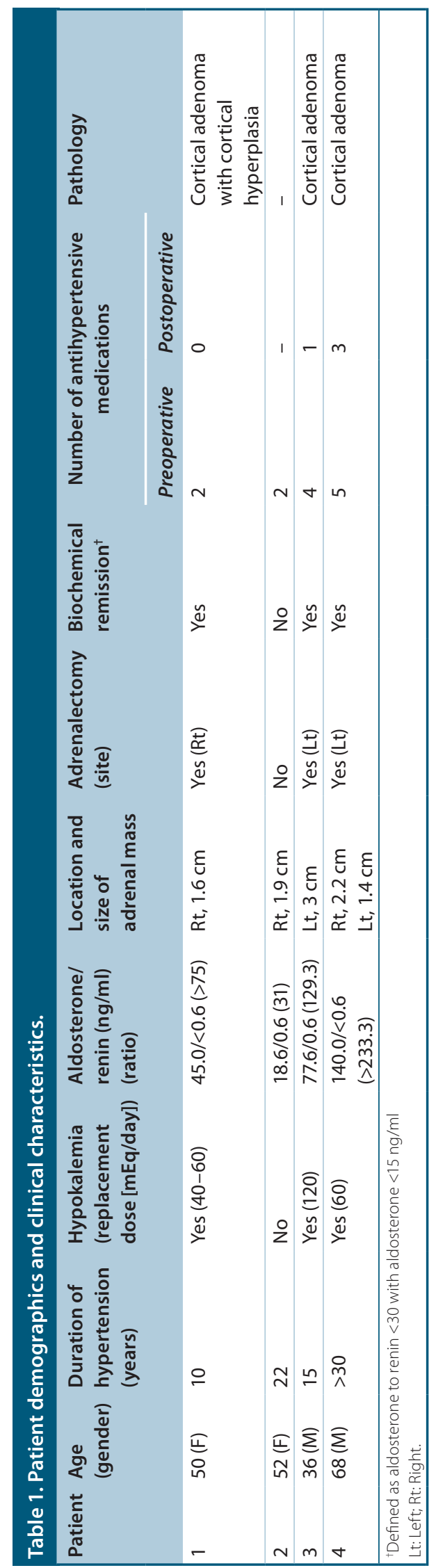




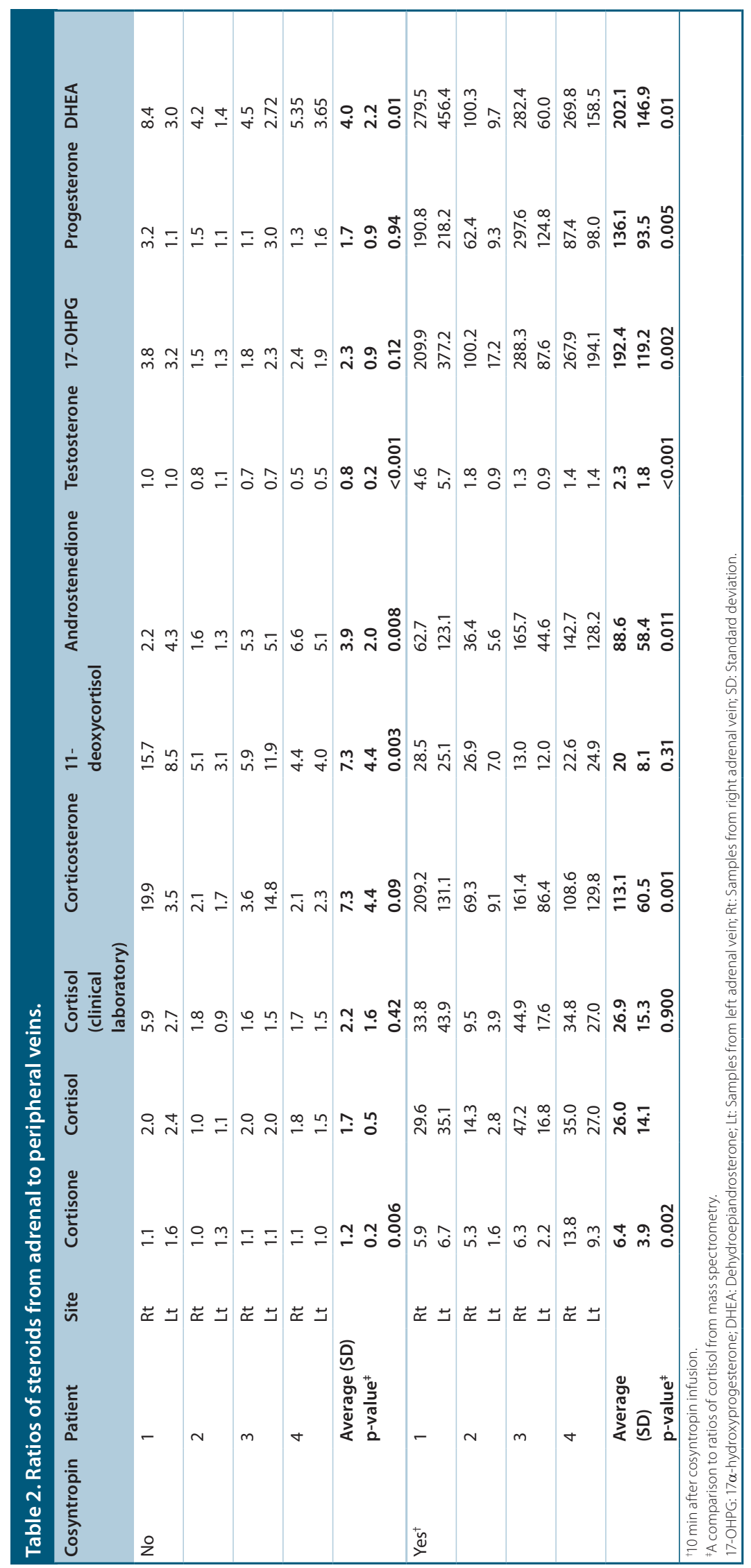

\section{- Statistical analysis}

Fisher's exact test was used to compare categorical variables [17]. Student's $t$-test was used to compare the mean of normally distributed continuous variables [18]. We used a successful adrenal vein catheterization, defined by the ratios of steroids from adrenal to peripheral veins being $>3$, without cosyntropin infusion, as a primary outcome. A two-tailed p-value $<0.05$ was considered statistically significant. Statistical analysis was performed using SPSS $^{\circledR}$ v21.0 for Windows (SPSS, Inc., IL, USA).

\section{Results}

There were three patients with PA due to a unilateral hyperfunctioning adrenal gland and one patient with bilateral adrenal disease enrolled in this study. Patient demographics and clinical characteristics are summarized in Table 1. All three patients with unilateral disease underwent a laparoscopic adrenalectomy without complications. These patients were in biochemical remission and did not require potassium replacement therapy. One patient did not require antihypertensive medication, and two patients required fewer antihypertensive medications. Although we recommended spironolactone to the patient with bilateral adrenal disease, the patient continued to receive two antihypertensive medications (hydralazine and verapamil).

The ratios of steroids from adrenal to peripheral veins both before and after cosyntropin infusion are summarized in Table 2 \& Figure 1. Compared with the mean ratio of cortisol prior to cosyntropin infusion, those of 11-deoxycortisol (7.3 vs 1.7, $\mathrm{p}=0.008$ ), androstenedione (3.9 vs $1.7, \mathrm{p}=0.008)$ and DHEA (4.0 vs 1.7 , $\mathrm{p}=0.01)$ were significantly higher. When the ratio of $>3$ prior to cosyntropin infusion was used to indicate a successful catheterization, none of the ratios $(\mathrm{n}=8)$ of cortisol from adrenal to peripheral veins analyzed by LC-MS/MS exceeded 3:1, while all the ratios of 11-deoxycortisol $(\mathrm{p}<0.001)$ did. Seventy-five percent of ratios of DHEA $(n=6 / 8)$ and $62.5 \%$ of ratios of androstenedione $(\mathrm{n}=5 / 8, \mathrm{p}=0.026)$ were $>3$. We also observed significantly lower mean ratios of cortisone (1.2 vs $1.7, \mathrm{p}=0.006)$ and testosterone ( 0.8 vs $1.7, \mathrm{p}<0.001)$. We found no significant difference when we compared the ratios of $17-$ OHPG $(\mathrm{p}=0.12)$, progesterone $(\mathrm{p}=0.94)$ and cortisol analyzed by a clinical laboratory ( $p=0.42)$ to those of cortisol analyzed by mass spectrometry. 

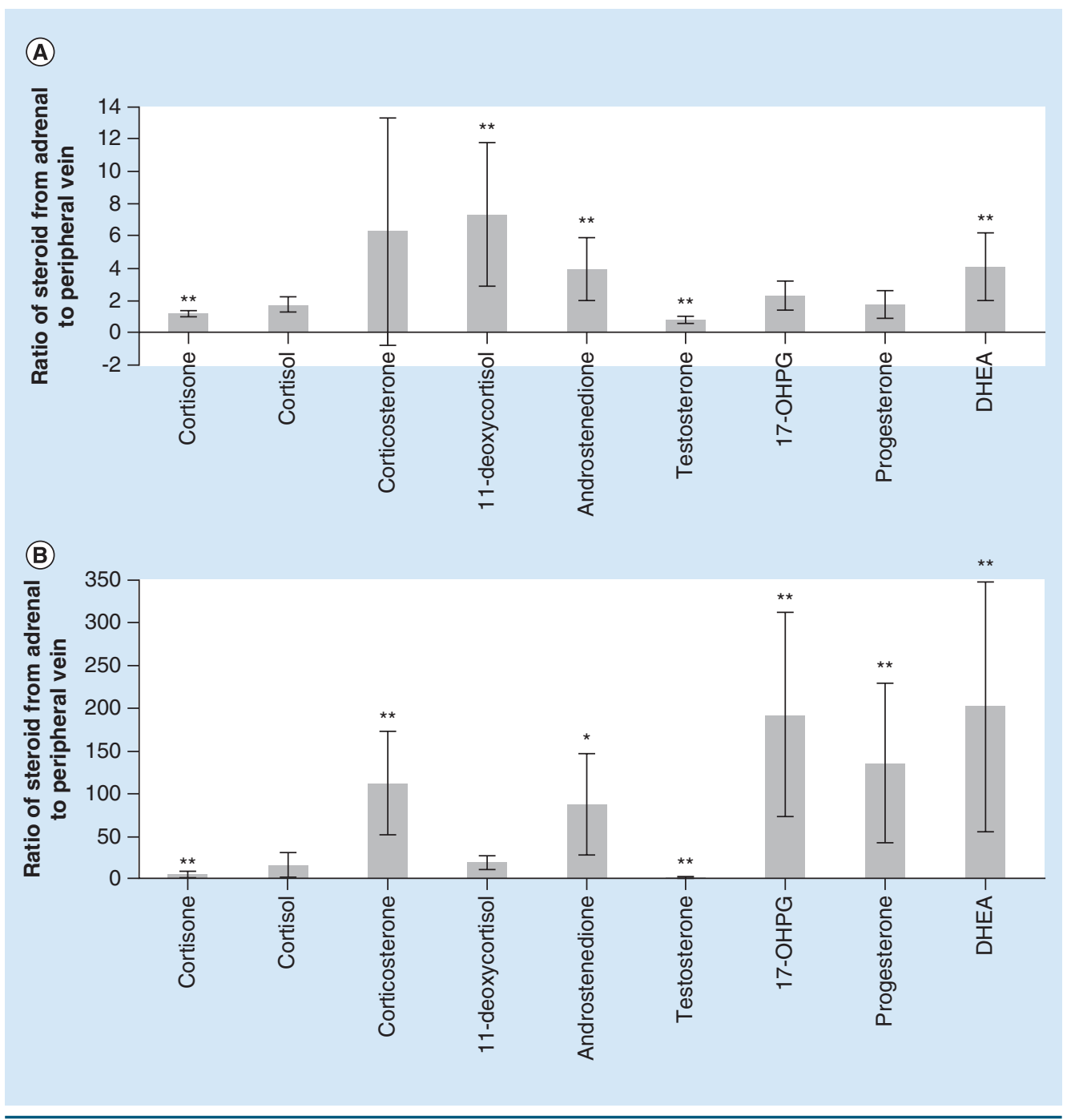

Figure 1. The ratio of steroids from adrenal vein to peripheral vein with and without cosyntropin. The ratios of steroids from adrenal to peripheral veins before (A) and after cosyntropin infusion (B). The comparisons were made to the ratio of cortisol.

${ }^{*} \mathrm{p}<0.05 ;{ }^{* *} \mathrm{p}<0.01$.

17-OHPG: 17 $\alpha$-hydroxyprogesterone; DHEA: Dehydropandrosterone.

Compared with the mean ratio of cortisol $10 \mathrm{~min}$ after cosyntropin infusion, those of corticosterone (113.1 vs $60.5, \mathrm{p}=0.001$ ), androstenedione (88.6 vs 26.0, $\mathrm{p}=0.011), 17-\mathrm{OHPG}$ (192.4 vs 26.0, $\mathrm{p}=0.002$ ), progesterone (136.1 vs $26.0, \mathrm{p}=0.005)$ and DHEA (202.1 vs 26.0, $\mathrm{p}=0.01$ ) were significantly higher. When the ratio of $>5$ after cosyntropin infusion was used to indicate a successful adrenal vein catheterization, there was no difference between the rate of successful catheterization when we compared the use of cortisol to corticosterone, 11-deoxycortisol, androstenedione, 17-OHPG, progesterone (87.5\% of cortisol $[n=7 / 8]$ vs $100 \%[n=8 / 8]$ of $\mathrm{p}=1.00)$ and DHEA. There was no difference in the mean ratios of cortisol analyzed by mass spectrometry to those of 11-deoxycortisol (0.31) and cortisol analyzed by a clinical laboratory $(\mathrm{p}=0.90)$.

The ratios of normalized aldosterone to steroids from dominant to nondominant adrenal glands before and after cosyntropin infusion are summarized in Table 3 \& Figure 2. Prior to cosyntropin infusion, the ratios of aldosterone normalized by each of the steroids in our patient with bilateral adrenal disease were all below 2 . The ratios in patients with unilateral disease prior to cosyntropin infusion were all $>2$ when 


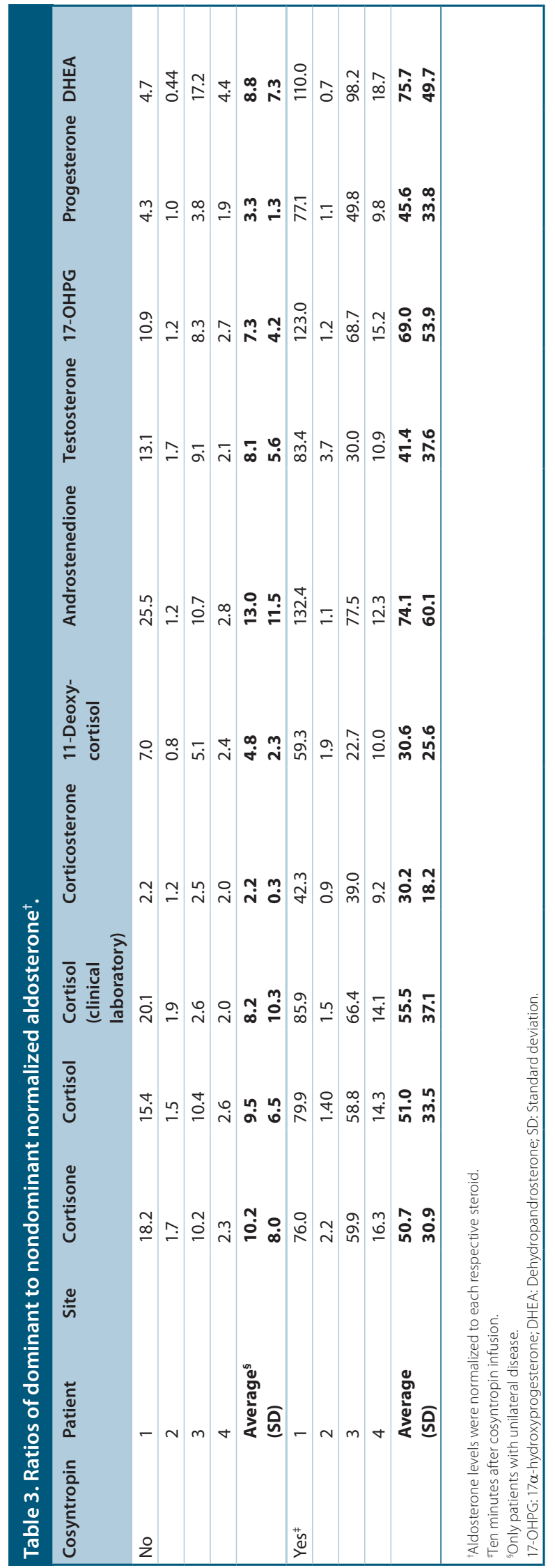

we normalized the aldosterone levels with cortisone, cortisol, 11-deoxycortisol, androstenedione, testosterone, 17-OHPG, progesterone and DHEA. Ten minutes after cosyntropin infusion, the ratios of aldosterone normalized by each of the steroids in the patient with bilateral adrenal disease were all below 4 . The ratios in patients with unilateral disease 10 min after cosyntropin infusion were all $>4$, regardless of the steroids used in normalization. All of the normalized ratios of aldosterone to cortisol and eight other steroids from nondominant adrenal glands in three patients with unilateral disease were lower than those of peripheral samples.

\section{Discussion}

PA is a common cause of hypertension, with a prevalence of $>10 \%$ in hypertensive patients [19]. PA causes a constellation of cardiovascular, renal and metabolic sequelae above and beyond the effects produced by high blood pressure alone [20]. Because AVS is considered the gold standard in the identification of patients with PA from unilateral hyperfunctioning adrenal glands who can benefit from adrenalectomy, a biochemical confirmation of successful adrenal vein catheterization is critically important to accurately interpret the results. In this study, we demonstrated that without cosyntropin infusion, cortisol is a poor marker for biochemically confirming the position of the catheter because none of the eight samples from adrenal veins had gradients three-times greater than those of peripheral veins. However, all samples from adrenal veins had more than three-times higher concentrations of 11-deoxycortisol than peripheral vein samples. This suggests that 11 -deoxycortisol is a more accurate marker than cortisol for biochemically confirming a correct catheter placement, without the need for cosyntropin infusion. In addition, 11-deoxycortisol was a good marker, comparable to cortisol, in identifying patients with unilateral adrenal disease because it correctly identified three patients with unilateral disease and one patient with bilateral disease with or without cosyntropin infusion. Although the ratios of corticosterone and androstenedione from adrenal to peripheral veins were significantly higher than those of cortisol, the ratios were inconsistent without cosyntropin infusion. There were no differences in the rates of successful adrenal vein catheterization after cosyntropin infusion between cortisol and corticosterone, 11-deoxycortisol and androstenedione. 


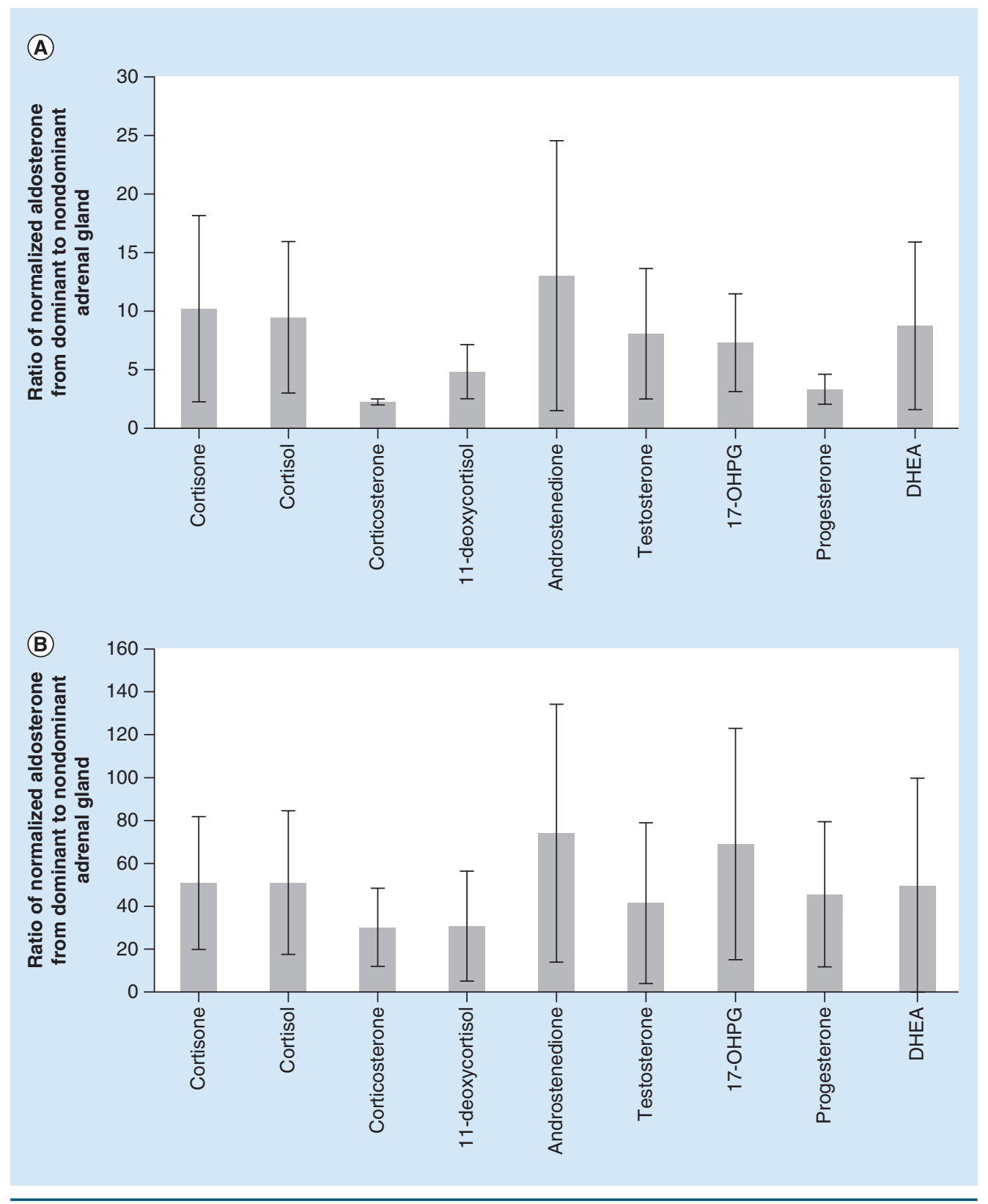

Figure 2. The ratio of aldosterone normalized by steroids from dominant gland to nondominant gland. The ratios of normalized aldosterone to steroids from dominant to nondominant adrenal glands before (A) and after cosyntropin infusion (B).

17-OHPG: 17 $\alpha$-hydroxyprogesterone; DHEA: Dehydropandrosterone.

AVS is a technically demanding procedure, especially the successful cannulation of small adrenal veins. Because the right adrenal vein is typically shorter and has direct drainage to the inferior vena cava, it is difficult to place and maintain the catheter tip in the right adrenal vein during the collection of samples. Thus, biochemical confirmation using adrenal produced steroids, such as cortisol, is mandatory. To correct for sample dilution by nonadrenal blood, the aldosterone level from AVS is normalized using cortisol because cortisol (and other steroids) production by both adrenal glands is presumed to be equal in patients with PA. However, we found that cortisol production from adrenal glands without stimulation by cosyntropin cannot be reliably used to indicate a successful adrenal vein catheterization, consistent with a 
previous report [15]. The use of cosyntropin to exaggerate the cortisol levels from the adrenal vein compared with peripheral cortisol levels has been considered to be a requirement to improve success rates of AVS [15,21]. We used a highly accurate LC-MS/MS to simultaneously assess the levels of cortisol and eight other steroids from AVS and identify 11-deoxycortisol as a superior marker compared with cortisol for biochemically confirming catheter position without cosyntropin infusion. To directly compare the performance of eight other steroids from the adrenal gland to cortisol, we applied the previously validated criteria for catheter positioning and lateralization [7]. The contralateral suppression of aldosterone secretion, defined as lower normalized aldosterone in a nondominant adrenal gland than that of a peripheral sample, was suggested to be a predictor for biochemical resolution of PA and improvement in blood pressure [22]. Thus, it has been used as additional criteria for identifying patients with unilateral disease when cosyntropin is not given [23]. We found the suppression of aldosterone secretion in all three patients with unilateral disease, regardless of steroids used in normalization. All had biochemical resolution of PA.

Our results suggest that cosyntropin infusion is not necessary if aldosterone levels are normalized with 11-deoxycortisol. The omission of cosyntropin can simplify and shorten the length of the procedure and reduce false-negative results for lateralization [24]. Thus, the use of 11-deoxycortisol may reduce the cost of AVS and increase the success rates as compared with the use of cortisol. The study by Peitzsch et al. focused on the use of LC-MS/MS to detect multiple steroids in AVS samples and compared the levels of these steroids from adrenal and peripheral vein samples to those of cortisol [25]. They found that median adrenal/peripheral ratios of 11-deoxycortisol, 17-OHPG, pregnenolone, androstenedione and DHEA were several times higher than those for cortisol. Eisenhofer et al. subsequently reported that LC-MS/MS-based steroid profiling during AVS had higher aldosterone lateralization ratios in patients with aldosterone-producing adenoma than immunoassays and suggested that multisteroid panel by LCMS/MS can identify patients with unilateral disease [26]. Although previous studies of steroid profiling in AVS samples of patients with PA demonstrated higher levels of 11-deoxycortisol and other steroids compared with cortisol $[25,26]$, no comparison was made to directly compare the performance of these markers to that of cortisol in confirming catheter position in the absence of cosyntropin. Although cortisol is one of the most abundantly produced adrenal steroids, the lower adrenal to peripheral ratio compared with that of 11-deoxycortisol and other steroids can be explained by the relatively slower clearance of cortisol because most circulating cortisol is bound to globulin $[27,28]$. Despite our small sample size, we were able to demonstrate a significant difference in the performance between cortisol and other steroids as markers indicating a successful adrenal vein catheterization. However, because this work is preliminary, further validation in larger cohorts is warranted.

In summary, we demonstrated in a pilot study that the ratios of corticosterone, 11-deoxycortisol, androstenedione and DHEA from adrenal to peripheral veins were significantly higher than that of cortisol without cosyntropin infusion. The ratios of 11-deoxycortisol from adrenal to peripheral veins in all samples without cosyntropin infusion from patients with unilateral disease indicated successful catheterizations, while none of the ratios using cortisol indicated successful adrenal vein catheterization. There was no difference in identifying patients with unilateral adrenal disease when we compared 11-deoxycortisol to cortisol.

In conclusion, this pilot study suggests that 11-deoxycortisol is potentially a superior marker compared with cortisol in confirming a successful adrenal vein catheterization without cosyntropin infusion while achieving the same lateralization performance. This finding warrants validation in larger cohort studies.

Financial \& competing interests disclosure

The research activities performed in this manuscript were supported by the Intramural Research Program of the Center for Cancer Research, National Cancer Institute, National Institutes of Health (grant ID 8014165). The authors have no other relevant affiliations or financial involvement with any organization or entity with a financial interest in or financial conflict with the subject matter or materials discussed in the manuscript apart from those disclosed.

No writing assistance was utilized in the production of this manuscript.

\section{Ethical conduct of research}

The authors state that they have obtained appropriate institutional review board approval or have followed the 
principles outlined in the Declaration of Helsinki for all human or animal experimental investigations. In addition, for investigations involving human subjects, informed consent has been obtained from the participants involved.

\section{References}

1 Conn JW. Presidential address. I. Painting background. II. Primary aldosteronism, a new clinical syndrome. J. Lab. Clin. Med. 45(1), 3-17 (1955).

2 Mulatero P, Stowasser M, Loh KC et al. Increased diagnosis of primary aldosteronism, including surgically correctable forms, in centers from five continents. J. Clin. Endocrinol. Metab. 89(3), 1045-1050 (2004).

3 Mckenzie TJ, Lillegard JB, Young WF Jr, Thompson GB. Aldosteronomas-state of the art. Surg. Clin. North. Am. 89(5), 1241-1253 (2009).

4 Milliez P, Girerd X, Plouin PF, Blacher J, Safar ME, Mourad JJ. Evidence for an increased rate of cardiovascular events in patients with primary aldosteronism. J. Am. Coll. Cardiol. 45(8), 1243-1248 (2005).

5 Zarnegar R, Bloom AI, Lee J et al. Is adrenal venous sampling necessary in all patients with hyperaldosteronism before adrenalectomy? J. Vasc. Interv. Radiol. 19(1), 66-71 (2008).

6 Mathur A, Kemp CD, Dutta U et al. Consequences of adrenal venous sampling in primary hyperaldosteronism and predictors of unilateral adrenal disease. J. Am. Coll. Surg. 211(3), 384-390 (2010).

7 Webb R, Mathur A, Chang R et al. What is the best criterion for the interpretation of adrenal vein sample results in patients with primary hyperaldosteronism? Ann Surg. Oncol. 19(6), 1881-1886 (2012).

8 Young WF, Stanson AW, Thompson GB, Grant CS, Farley DR, Van Heerden JA. Role for adrenal venous sampling in primary aldosteronism. Surgery 136(6), 1227-1235 (2004).

9 Gordon RD. Primary aldosteronism. J. Endocrinol. Invest. 18(7), 495-511 (1995).

10 Kline GA, Harvey A, Jones C et al. Adrenal vein sampling may not be a gold-standard diagnostic test in primary aldosteronism: final diagnosis depends upon which interpretation rule is used. Variable interpretation of adrenal vein sampling. Int. Urol. Nephrol. 40(4), 1035-1043 (2008).
11 Vonend O, Ockenfels N, Gao X et al. Adrenal venous sampling: evaluation of the German Conn's registry. Hypertension 57(5), 990-995 (2011).

12 Nwariaku FE, Miller BS, Auchus R et al. Primary hyperaldosteronism: effect of adrenal vein sampling on surgical outcome. Arch. Surg. 141(5), 497-502; discussion 502-493 (2006).

13 Young WF, Stanson AW. What are the keys to successful adrenal venous sampling (AVS) in patients with primary aldosteronism? Clin. Endocrinol. 70(1), 14-17 (2009).

14 Seccia TM, Miotto D, De Toni R et al. Adrenocorticotropic hormone stimulation during adrenal vein sampling for identifying surgically curable subtypes of primary aldosteronism: comparison of 3 different protocols. Hypertension 53(5), 761-766 (2009).

15 Kline GA, So B, Dias VC, Harvey A, Pasieka JL. Catheterization during adrenal vein sampling for primary aldosteronism: failure to use (1-24) ACTH may increase apparent failure rate. J. Clin. Hypertens. (Greenwich) 15(7), 480-484 (2013).

16 Stolze BR, Gounden V, Gu J et al. An improved micro-method for the measurement of steroid profiles by APPI-LC-MS/MS and its use in assessing diurnal effects on steroid concentrations and optimizing the diagnosis and treatment of adrenal insufficiency and CAH. J. Steroid Biochem. Mol. Biol. 162, 110-116 (2016).

17 Fisher RA. On the interpretation of $\chi^{2}$ from contingency tables, and the calculation of $\mathrm{p}$. J. R. Stat. Soc. 85(1), 87-94 (1922).

18 Student. The probable error of a mean. Biometrika 6(1), 1-25 (1908).

19 Funder JW, Carey RM, Fardella C et al. Case detection, diagnosis, and treatment of patients with primary aldosteronism: an endocrine society clinical practice guideline. J. Clin Endocrinol. Metab. 93(9), 3266-3281 (2008).

20 Rossi GP, Sechi LA, Giacchetti G, Ronconi V, Strazzullo P, Funder JW. Primary aldosteronism: cardiovascular, renal and metabolic implications. Trends Endocrin. Metab. 19(3), 88-90 (2008).

21 Monticone S, Viola A, Rossato D et al. Adrenal vein sampling in primary aldosteronism: towards a standardised protocol. Lancet Diabetes Endocrinol. 3(4), 296-303 (2015).

22 Wolley MJ, Gordon RD, Ahmed AH, Stowasser M. Does contralateral suppression at adrenal venous sampling predict outcome following unilateral adrenalectomy for primary aldosteronism? A retrospective study. J. Clin. Endocrinol. Metab. 100 (4), 1477-1484 (2015).

23 Rossi GP, Auchus RJ, Brown M et al. An expert consensus statement on use of adrenal vein sampling for the subtyping of primary aldosteronism. Hypertension 63(1), 151-160 (2014).

24 Rossi GP, Pitter G, Bernante P, Motta R, Feltrin G, Miotto D. Adrenal vein sampling for primary aldosteronism: the assessment of selectivity and lateralization of aldosterone excess baseline and after adrenocorticotropic hormone (ACTH) stimulation. J. Hypertens. 26(5), 989-997 (2008).

25 Peitzsch M, Dekkers T, Haase M et al. An LC-MS/MS method for steroid profiling during adrenal venous sampling for investigation of primary aldosteronism. J. Steroid Biochem. Mol. Biol. 145, 75-84 (2015).

26 Eisenhofer G, Dekkers T, Peitzsch M et al. Mass spectrometry-based adrenal and peripheral venous steroid profiling for subtyping primary aldosteronism. Clin. Chem. 62(3), 514-524 (2016).

27 Bright GM. Corticosteroid-binding globulin influences kinetic parameters of plasma cortisol transport and clearance. J. Clin. Endocrinol. Metab. 80(3), 770-775 (1995).

28 Perogamvros I, Aarons L, Miller AG, Trainer PJ, Ray DW. Corticosteroid-binding globulin regulates cortisol pharmacokinetics. Clin. Endocrinol. 74(1), 30-36 (2011). 